\title{
Questes
}

Revue pluridisciplinaire d'études médiévales

$13 \mid 2008$

Figures royales à l'ombre du mythe

\section{Les Neuf Preux : entre édification et glorification}

\section{Anne Salamon}

\section{OpenEdition}

\section{Journals}

Édition électronique

URL : http://journals.openedition.org/questes/1527

DOI : 10.4000/questes. 1527

ISSN : 2109-9472

\section{Éditeur}

Les Amis de Questes

\section{Édition imprimée}

Date de publication : 15 janvier 2008

Pagination : 38-52

ISSN : 2102-7188

\section{Référence électronique}

Anne Salamon, «Les Neuf Preux : entre édification et glorification», Questes [En ligne], 13 | 2008, mis en ligne le 01 janvier 2014, consulté le 02 mai 2019. URL : http://journals.openedition.org/ questes/1527 ; DOI : 10.4000/questes. 1527 


\title{
Les Neuf Preux : entre édification et glorification
}

\author{
Anne SAlamon
}

Le motif littéraire et esthétique des Neuf Preux connaît une immense fortune en Europe entre le XIV ${ }^{\mathrm{e}}$ et le XVI $\mathrm{X}^{\mathrm{e}}$ siècle. La liste des Neuf Preux, composée de «rois» historiques ou pseudo-historiques, s'organise en trois triades, la première, biblique (Josué, David, Judas Macchabée), la deuxième, païenne (Hector, Alexandre, Jules César), et la dernière, chrétienne (Arthur, Charlemagne, Godefroy de Bouillon). Fixée sous sa forme canonique dans les Voux du Paon de Jacques de Longuyon vers 1310-1312, cette liste s'est répandue sous des formes et sur des supports extrêmement variés. Même si, pour nous, la présence de certains noms dans cette liste semble moins évidente, le succès au Moyen Âge de chacune de ces figures prises individuellement est incontestable. Héros populaires, modèles de chevalerie, les Neuf Preux apparaissent dès les Vœux du Paon comme des incarnations de l'idéal chevaleresque et la cristallisation d'une certaine idéologie nobiliaire. Le statut ambigu de ces personnages aux frontières de l'histoire, de la légende et de la fiction ainsi que la richesse de leur représentation au Moyen Âge confèrent à leur mise en série une signification particulière, la liste des Neuf Preux se trouvant dotée d'une forte dimension mythique. Nous nous attacherons ici à étudier ce que représente dans l'imaginaire de l'époque la réunion de ces neuf héros, figures tirées de textes et de genres si différents, pour ensuite examiner les différentes utilisations faites de ce motif et tout particulièrement son rapport aux problématiques de la gloire et de l'exemplarité. 


\section{Constitution d'un motif de cour}

La liste des Neuf Preux permet l'évocation de tout un pan de la littérature narrative médiévale. Elle entretient un rapport étroit avec la Bible, dont proviennent les trois premiers Preux, mais aussi avec l'épopée et surtout avec les romans de chevalerie et la littérature romanesque courtoise, que l'on songe aux romans antiques pour Alexandre et Hector ou à la matière de Bretagne pour Arthur. Une certaine ambiguïté apparaît d'emblée quant au statut des Preux. En effet, c'est incontestablement l'immense succès de ces romans et œuvres de fiction où ils apparaissent qui assure le fondement de la faveur des Neuf Preux, bien que ce motif soit, à la fin du Moyen Âge, dans de nombreux cas, attaché à un contexte historique. Dès la Chanson de Roland, le terme de "preux » est associé au terme «courtois» pour qualifier Olivier ${ }^{1}$, attestant d'une sorte de perfection du chevalier qui associe des qualités sociales et morales à des qualités guerrières $^{2}$. Pourtant, le motif des Preux n'est pas un thème de littérature courtoise au sens propre du terme ; si cela avait été le cas, on se serait attendu à trouver parmi eux Lancelot ou Gauvain, plutôt qu'Arthur. Glynnis M. Cropp ${ }^{3}$, dans son analyse des vers sur les Preux tirés des Vœux $d u$ Paon, constate que « les faits ont été choisis pour faire ressortir l'idéal de la chevalerie et l'image du conquérant militaire, qui est aussi roi ou prince souverain ${ }^{4}$. En effet, chaque strophe est composée d'une rapide biographie situant chaque héros chronologiquement et géographiquement et rappelant quelques hauts faits constituant des lieux communs attachés à chaque personnage. Dans la constitution du panthéon héroïque que se veut la liste des Neuf Preux, la préoccupation n'est plus la même en cette fin du Moyen Âge que dans la

\footnotetext{
${ }^{1}$ La Chanson de Roland, éd. Cesare SEGRE, Genève, Droz, 2003, v. 576 et 3755.

${ }^{2}$ Voir à ce sujet Dictionnaire du Moyen Âge, Claude GAUVARD, Alain DE LIBERA, Michel ZINK (dir.), Paris, PUF, 2002, « courtoisie », p. 360.

${ }^{3}$ Glynnis M. CROPP, « Les vers sur les Neuf Preux », Romania, t. 120, 2002, p. 449-482.

${ }^{4}$ Ibid., p. 458.
} 
littérature courtoise; le corollaire de la prouesse n'est plus l'amour mais la gloire en elle-même.

À l'origine du motif, les Neuf Preux sont ainsi des rois ou princes à la valeur militaire et guerrière exceptionnelle. Dans les trois compilations de la fin $\mathrm{du} \mathrm{XV}^{\mathrm{e}}$ siècle consacrées aux Preux, on a affaire à neuf « souverains » qui ont marqué l'histoire et qui doivent être loués pour leur exemplarité, dans une optique proche de celle des miroirs au prince, dans la mesure où elles présentent un texte narratif historique, destiné à un roi, un prince ou un grand seigneur, qui vise à faire réfléchir sur le destin des royaumes. C'est cette conception de l'histoire comme succession de hauts faits qu'exprime Froissart dans le prologue des Chroniques quand il écrit :

«Afin que les grans merveilles et li biau fait d'armes qui sont avenu par les grans guerres de France et d'Engleterre et des royaumes voisins, dont li roy et leurs consaulz sont cause, soient notablement registré et, ou temps present et à venir, veu et congneu, je me voel ensonniier de l'ordonner et mettre en prose selonch le vraie information que j'ay eu des vaillans hommes, chevaliers et escuiers, qui les ont aidiés à acroistre, et ossi de aucuns rois d'armes et leurs mareschaus, qui par droit sont et doient estre juste inquisiteur et raporteur de tels besongnes. ${ }^{5}$

Gloire, honneur et réussite aux armes sont le sujet de l'histoire, ce que confirme la place importante dans le processus de l'écriture historique des hérauts et rois d'armes, experts en cette matière. La question des hérauts d'armes touche de près la problématique des Neuf Preux et de la chevalerie. En effet, les XIV ${ }^{\mathrm{e}}$ et $\mathrm{XV}^{\mathrm{e}}$ siècles constituent une période de mutation du point de vue militaire et voient, avec l'évolution du matériel et des techniques de combat, le déclin de la chevalerie sur le champ de bataille. Le culte des héros intervient dans ce contexte d'entretien factice de la courtoisie dans des manières et des jeux de cour où les valeurs chevaleresques ne constituent plus que de vagues idéaux,

\footnotetext{
${ }^{5}$ Jean Froissart, Chroniques, éd. Siméon LuCE, Paris, $\mathrm{M}^{\mathrm{me}} \mathrm{V}^{\mathrm{e}}$ Jules Renouard, 1869, t. 1, Prologue, p. 1.
} 
souvenirs d'un passé idéalisé. Les divertissements de la noblesse et de la cour, qui sont imprégnés de ces idées, consistent alors en une imitation de ces héros ; le Banquet du Faisan ${ }^{6}$ ou les autres fêtes grandioses tenues à la cour de Bourgogne, ne sont pas sans rappeler les banquets du Perceforest ${ }^{7}$, tandis que les tournois sont censés évoquer un cadre romanesque, comme par exemple le tournoi de la «Joyeuse Garde» organisé par René d'Anjou. Ce même René d'Anjou, quand il va rendre hommage à la dépouille mortelle de Charles le Téméraire, «avoit une grant barbe d'or venant jusques à la seinture en significacion des anciens preux... $»^{8}$ et François $\mathrm{I}^{\mathrm{er}}$ aimait encore à s'habiller à la manière de l'un des Preux ${ }^{9}$, tandis que tout au long des $X V^{e}$ et $X^{e}$ siècle le cortège des Preux et des Preuses constitue l'un des thèmes récurrents des entrées royales ${ }^{10}$.

Les Neuf Preux sont enracinés dans la vie et la culture nobiliaire du temps et leur représentation est omniprésente sur toutes sortes de supports, constituant l'un des motifs décoratifs préférés des demeures de la noblesse. Il serait impossible ici de faire un inventaire complet de toutes les apparitions de ce motif. On pourra toutefois citer la présence de plusieurs tapisseries des Preux dans les collections des ducs de Bourgogne ${ }^{11}$, les différentes salles dites « des Preux » dans les châteaux de Langeais ${ }^{12}$, de la $\operatorname{Manta}^{13}$ ou de $\mathrm{Coucy}^{14}$, qui

\footnotetext{
${ }^{6}$ Voir Georges Doutrepont, La littérature française à la cour des ducs de Bourgogne: Philippe le Hardi, Jean sans Peur, Philippe le Bon, Charles le Téméraire, Paris, Champion, 1909, p. 104 sqq.

${ }^{7}$ Voir Le Roman de Perceforest, éd. Gilles Roussineau, I İre partie, Genève, Droz, 2007, p.xxxiv sqq.

${ }^{8}$ Journal de Jean de Roye connu sous le nom de Chronique scandaleuse, éd. Bernard DE MANDROT, Paris, Renouard, 1896, t. II, p. 42.

9 Johan HulzIngA, L'automne du Moyen Âge, Paris, Payot, 2002 (édition originale Herfsttij der Middeleeuwen, Haarlem, 1919), p. 113.

${ }^{10}$ Bernard GuENÉE et Françoise LEHOUX, Les entrées royales françaises de 1328 à 1515 , Paris, CNRS, 1968.

${ }^{11}$ Georges DOUTREPONT, op. cit., p. 117.

12 Un rêve de chevalerie: les Neuf Preux, exposition du château de Langeais, 2 mars-3 novembre 2003, Jean FAVIER (dir.), Paris, Institut de France, 2003.

13 Paolo D’AnConA, «Gli affreschi del castello di Manta nel Saluzzese », L'Arte, t. VIII, I, 1905, p. 94-106 et 184-198.
} 
doivent leurs noms respectivement à des tapisseries, des fresques et des statues représentant les Preux, ou encore le fait que chacune des neuf tours des châteaux de Pierrefonds et de Vincennes portent le nom d'un Preux. Cependant, les Preux sont encore plus étroitement associés aux éléments constitutifs de cette vie chevaleresque de la fin du Moyen Âge que sont les tournois, l'héraldique et les ordres de chevalerie; nous laisserons de côté le succès des tournois, qui trouvaient principalement leur inspiration dans les romans de chevalerie. Les hérauts ont attribué très tôt des armes aux Neuf Preux, quand ils n'en avaient pas déjà (les armoiries d'Arthur, pour ne prendre qu'un exemple, constituées de trois couronnes sur un fond azur, existaient déjà au XIII ${ }^{\mathrm{e}}$ siècle dans les romans puis dans les enluminures ${ }^{15}$ ); par ailleurs, dans les traités de blason $\mathrm{du} \mathrm{XV}^{\mathrm{e}}$, mais surtout $\mathrm{du} \mathrm{XVI}^{\mathrm{e}}$ siècle, Jules César, Alexandre ou encore les Neuf Preux dans leur ensemble sont considérés comme les fondateurs de l'art héraldique ${ }^{16}$. Ils sont également liés aux ordres de chevalerie - des dessins coloriés à l'image des Preux et des Preuses accompagnent des documents relatifs à l'ordre de la Toison d'Or (ms. $1312 \mathrm{du}$ fonds Clairambault de la $\mathrm{BNF}^{17}$ ) - mais surtout à leurs règlements. En effet, les statuts de l'ordre de la Jarretière et ceux de l'ordre de l'Etoile, datant tous deux de la moitié du XIV siècle, prévoient que, tous les ans, trois princes, trois barons et trois chevaliers seraient assis à une table d'honneur en récompense de leur excellence aux armes, tandis qu'à l'origine, la Jarretière

\footnotetext{
14 Gianni Mombello, «Les complaintes des .IX. Malheureux et des .IX. Malheureuses. Variations sur le thème des Neuf Preux et du "Vado Mori " ", Romania, t. LXXXVII, 1966, p. 345-378, particulièrement p. 352.

${ }^{15}$ Michel PAstoureau, Les chevaliers de la table ronde, Lathuile, Editions du Gui, 2006, p. 34.

${ }^{16}$ Voir Gianni Mombello, art. cit., p. 357-358, et Claire BoudreAu, « Traités de blason et armoriaux : pédagogie et mémoire », dans Les armoriaux, Cahier du Léopard d'or, vol. 8, 1997, p. 383-390, et plus particulièrement p. 388-389.

${ }^{17}$ Gianni Mombello, art. cit, p. 356.
} 
devait être attribuée de la même manière parmi un groupe de neuf ainsi constitué. $^{18}$

L'importance accordée par la noblesse au thème des Preux peut être résumée par cette réflexion de Froissart dans le prologue des Chroniques :

« Mais ainçois que j'en commence à parler, je voel un petit tenir et demener le pourpos de proèce, car c'est une si noble vertu, et de si grant recommandation, que on ne le doit mies passer trop briefment, car elle est mère materièle et lumière des gentilz hommes, et, si com la busce ne poet ardoir sans feu, ne poet li gentilz homs tenir à parfaite honneur ne à le glore dou monde, sans proèce. $»^{19}$

Cette primauté de la prouesse dans la constitution du "gentilhomme», de l'homme noble, explique la faveur du groupe constitué par les Neuf Preux, liste fondée à l'origine sur la popularité de chaque personnage individuellement, mais qui, considérée dans son ensemble, constitue une sorte de best of en matière de prouesse et de gloire. L'utilisation du motif dans les textes va donc être orientée par deux objectifs, l'exemplification et la glorification.

\section{Emplois littéraires du motif des Neuf Preux}

Dans les Voux du paon, les Neuf Preux sont présentés comme des modèles de chevalerie, des souverains victorieux, références à l'aune desquelles peut être mesurée la valeur d'un chevalier, Porrus, qui surpasse leurs exploits lors d'une bataille. Glynnis M. Cropp montre l'articulation au cœur du motif entre d'une part «l'emploi rhétorique de l'exemplification comme moyen d'amplification » et d'autre part « la conception de l'histoire vue comme une série de hauts faits et d'accomplissements ». Les différentes utilisations possibles de la liste des Preux vont dépendre de ces deux caractéristiques. La

\footnotetext{
18 Voir Werner PARAVICINI, «Armoriaux et histoire culturelle: Le rôle d'armes des " Meilleurs Trois " ", dans Les armoriaux, Cahier du Léopard d'or, vol. 8, 1997, p. 361-381 et plus particulièrement p. 372.

19 JeAn Froissart, op.cit., t. 1, Prologue, p. 2.
} 
forme de la liste close et fixe confère une légitimité encore plus importante à ces figures d'autorité, ce que Jacqueline Cerquiglini ${ }^{20}$ a montré en étudiant en quoi la liste de noms constituait une «cristallisation de renom ${ }^{21}$; la liste close structurée autour d'un chiffre symbolique fonctionne comme un «véritable cadre de pensée $»^{22}$. D'autres tentatives ont été faites pour constituer des listes des meilleurs chevaliers, qui n'ont pas connu le même succès. Ainsi l'Armorial de Gerle $^{23}$ (manuscrit achevé au plus tard au tout début $\mathrm{du} \mathrm{XV}^{\mathrm{e}}$ siècle) aussi connu sous le nom de Rôle d'armes des «Meilleurs Trois» est constitué de quatorze triades. La symétrie et l'équilibre de la liste en trois fois trois (le chiffre trois est évidemment hautement symbolique) donnent une idée de totalité et de perfection en regroupant les trois meilleurs de chaque foi (païenne, juive et chrétienne, dans cet ordre le plus souvent) et de chaque époque, ce qui permet d'englober tous les types de vaillance et de gloire et toute l'histoire humaine depuis la création, en relation avec un progrès théologique de l'Histoire.

Toutefois, selon le type de texte et la place attribuée au motif, énumération de noms ou apparition plus détaillée, sa signification peut varier. Le premier type est assez peu représenté puisqu'il ne comporte que trois textes, mais il correspond à l'emploi le plus développé du thème des Preux dans de vastes compilations qui leur sont consacrées. L'Histoire des Neuf Preux et des Neuf Preues ${ }^{24}$ a été composée par Sébastien Mamerot entre 1460 et 1467 à la demande de son seigneur Louis de Laval ; la deuxième compilation, le Triumphe des Neuf Preux, anonyme, est imprimée pour la première fois en 1487 et la

\footnotetext{
${ }^{20}$ Jacqueline Cerquiglini, «Fama et les Preux : nom et renom à la fin du Moyen Âge », Médiévales 24, printemps 1993, p. 35-44.

${ }^{21}$ Ibid., p. 35.

${ }^{22}$ Ibid., p. 36.

${ }^{23}$ Sur cet armorial, voir Warner PARAVICINI, art. cit.

${ }^{24}$ Je prépare actuellement dans le cadre de ma thèse une édition critique de ce texte, encore inédit.
} 
troisième ne nous est parvenue que dans un manuscrit du XVIII ${ }^{\mathrm{e}} \operatorname{siècle}^{25}$, vraisemblablement une copie d'un manuscrit du $\mathrm{XV}^{\mathrm{e}}$ siècle.

Le second type d'occurrence, dont nous traiterons d'abord, consiste en une énumération des noms des Preux, qu'il s'agisse d'une liste sèche ou à laquelle s'ajoutent quelques faits majeurs et prototypiques et correspond en général aux apparitions du motif en poésie, illustrant ce que Jacqueline Cerquiglini nomme une «cristallisation de renom »: le nom seul constitue une garantie de gloire et d'autorité. Ce phénomène ne peut fonctionner que parce que ces noms constituent, à l'origine, un «pro-récit », c'est-à-dire que le nom seul suffit à évoquer tout un ensemble de données narratives ${ }^{26}$. Toutefois, avec la constitution d'une liste canonique des Neuf Preux, les noms, qui renvoyaient à différentes histoires, finissent par fonctionner comme un tout, formant une structure presque vide entièrement tournée vers la dénotation de la gloire, comme ici chez Eustache Deschamps ${ }^{27}$ (Balade XCIII, v. 1-20) :

Venez a moy, li hault prince ancien, .IX. hommes preux, et .IX. femmes de terre, Trois Sarrasin, trois Juif, trois Crestien : Hector le fort, Alixandre a conquerre, Julles Cesar, alez Josué querre, David aussi, Judas Machabeus, Charlemaine, Godefroy et Arthus Pour traictié faire entre le Franc et l'Angle, Car par eulx deux sont mains païs perdus :

Des or fust temps d'avoir paix, ce me semble.

Semiramis avecques ces preux vien, Deyphile, Marsopye o lui erre, Synoppe apres, Panthasilee tien, Tantha que j'aim, va Thamaris requerre, Yppolite, Menalope desserre,

\footnotetext{
${ }^{25}$ BNF f. fr. 12598 ; voir Richard TRACHSLER, Clotures du cycle arthurien, p. 298.

${ }^{26}$ Je renvoie ici aux pages que Richard Trachsler consacre à ce concept dans DisjointuresConjointures, Tübingen et Bâle, Francke, 2000, p. 25 sqq.

${ }^{27}$ Eustache Deschamps, Euvres complètes, éd. du Marquis DE Queux De SaINT-Hilaire, Paris, Firmin Didot, 1878, t. I, p. 199.
} 
Toutes et touz .XVIII. saillez sus, Mettez raison et le droit au dessus, Et ne veuillez soustenir le triangle. Qui tort ara, monstrez li voz vertus :

Des or fust temps d'avoir paix, ce me semble.

Quand chaque Preux est accompagné d'une rapide biographie ou de quelques faits marquants, il s'agit souvent d'une doxa le concernant, une série de clichés qui permettent une identification facile, comme c'est le cas dans les Voux $d u$ Paon, ici pour Hector (v. 7484-7494) $)^{28}$ :

Voirs est qu'Ector fu preus desmesureement

Car, si com li poëte nous vont ramentevant, Quant li roys Menelaus o son efforcement

Vint assegier a Troies le noble roy Priant

Pour Elayne sa fenme qu'il amoit durement

Que Paris ot ravie ains cel assemblement, Hector de la cité pris le gouvernement ;

Es issues c'on fist par son enortement

Ocist .XIX. roys sus son cors deffendant

Et amiraus et contes, ce croi je, plus de .C. :

Puis l'ocist Achillés moult trä̈teusement.

Le nom est évoqué comme garant, témoin d'un passé glorieux et en devient quasiment interchangeable. La liste dans son ensemble adopte un fonctionnement presque allégorique, sans toutefois que chaque Preux constitue une véritable entité abstraite ; comme l'explique Richard Trachsler :

« Il suffit de rappeler que certains personnages historiques ou littéraires peuvent incarner de façon emblématique des caractéristiques spécifiques et qu'ils deviennent ainsi l'exemplum ou le paradigma de quelque chose, c'est-à-dire qu'ils en viennent eux aussi à incarner un concept complexe,

\footnotetext{
${ }^{28} \mathrm{Je}$ cite ici l'édition récente des vers sur les Preux qu'a faite Glynnis M. CROPP, art. cit., p. 465-482. La seule édition des Voux du Paon est celle de R. L. RitchiE dans The Buik of Alexander or the Buik of the most noble and valiant Conquerour Alexander the Grit by John Barbour, Archdeacon of Aberdeen, Scottish Text Society, NS 12, 17, 21, 25, Edinbourg, Londres, 1921-1929. Les vers sur les Preux sont dans le volume 4, p. 402-406, vers 74847579 .
} 
comme Raison, Nature, etc. ce qui leur confère un statut ontologique hybride. $\|^{29}$

Du fait de ce statut intermédiaire, il n'est pas rare d'ailleurs que les Preux apparaissent, dans tout type de texte, en compagnie d'une figure allégorique, Dame Triomphe dans le Triumphe des Neuf Preux (compilation de la fin du $\mathrm{XV}^{\mathrm{e}}$ siècle) $)^{30}$, Honneur dans le Trosne d'Honneur ${ }^{31}$ de Jean Molinet ou encore Fortune dans le Chevalier Errant de Thomas III de Saluces ${ }^{32}$. Le Trosne d'Honneur, prosimètre composé par Jean Molinet à l'occasion de la mort de Philippe le Bon en 1467, est à cet égard tout à fait intéressant. Pour parvenir au trône d'Honneur, Philippe doit passer par neuf cieux où sont inscrites les neuf lettres qui composent son nom, Philippus, et où il est accueilli par une dame, allégorie d'une vertu qu'il a manifestée pendant sa vie et dont l'initiale correspond à chacune de ces lettres, et celui des Neuf Preux dont les actes ont le mieux illustré cette vertu. Ainsi, au premier ciel où est écrite la lettre P, Philippe est accueilli par Prudence et par Jules César « pour ce qu'il avoit tenu en grand honneur et chierté les preudhommes et saiges philosophes et lui meismes, par la gravité de son sens preadvisant choses futures, estoit parvenu a diademe imperial ${ }^{33}$. A l'issue de ce parcours, il est présenté par Vertu à Honneur, qui l'assoit à sa droite. Le texte construit un univers clos, permanent, par son architecture qui englobe tous les espaces (on songe aux neuf sphères du cosmos de Ptolémée) et toutes les vertus, dans lequel les Neuf Preux, figures exemplaires, constituent ainsi un échelon intermédiaire dans le processus d'abstraction, élevant l'homme mortel au niveau de l'allégorie qui représente, au-delà de la vie humaine, la permanence de l'honneur.

\footnotetext{
${ }^{29}$ Richard TRACHSLER, op. cit., p. 333.

${ }^{30}$ Ibid., p. 233-238 et id., Clôtures du cycle arthurien, Genève, Droz, 1996, p. 286-296.

${ }^{31}$ Les faitz et dictz de Jean Molinet, éd. Noël DupIRE, Paris, Société des Anciens Textes Français, 1936, t. I, p. 36-58.

32 Tommaso III di Saluzzo, Le Livre du Chevalier errant, éd. Marvin James WARD, Ph. D. University of North Carolina, 1984.

33 Les faitz et dictz de Jean Molinet, op. cit., p. 46.
} 
Les Neuf Preux sont donc également utilisés dans une stratégie de glorification, afin de louer un souverain, une lignée, un seigneur. Ces phénomènes (d'ailleurs observables dans tous les textes traitant des Preux sans se restreindre à la poésie), étudiés par Jacqueline Cerquiglini ${ }^{34}$, sont perceptibles dans les jeux opérés avec les noms de la liste, ajout ou substitution. La pratique la plus courante consiste à ajouter un dixième personnage, dont le nom est mis en série avec ceux des Neuf Preux. Qu'il soit explicitement cité comme dixième Preux, comme Du Guesclin chez Eustache Deschamps (ballades CCVII ${ }^{35}$, $\mathrm{CCCLXII}^{36}$ ) ou chez Sébastien Mamerot (même si la section consacrée à ce Preux, annoncée dans le prologue, n'a vraisemblablement jamais été composée), Pierre $\mathrm{I}^{\mathrm{er}}$ de Lusignan dans la Prise d'Alexandrie de Guillaume de Machaut ${ }^{37}$ ou encore Robert Bruce dans un poème écossais ${ }^{38}$, ou qu'un personnage apparaisse dans un rapport de comparaison avec les Neuf Preux, comme c'est le cas pour Porrus dans les Voeux du Paon ou pour Philippe de Bourgogne dans le Trosne d'honneur, la gloire accompagnant les Preux retombe sur lui. Dans le contexte troublé de la Guerre de Cent Ans et de la crise de la chevalerie à la fin du Moyen Âge, qu'il s'agisse de promouvoir un héros national ou de louer un seigneur et sa lignée, l'évocation des Neuf Preux permet de faire rejaillir leur gloire passée sur l'époque contemporaine. Cette volonté de louer un lignage est particulièrement visible dans le projet de Sébastien Mamerot d'ajouter Bertrand du Guesclin et Jeanne d'Arc à la liste des Preux et des Preuses. Le choix des deux personnages n'est pas en lui-même unique, mais une autre dimension s'ajoute dans le cas de la famille des Laval ${ }^{39}$. En effet, la grand-mère de Louis

\footnotetext{
${ }^{34}$ Jacqueline CERQUIGLINI, art. cit., p. 36-40.

${ }^{35}$ EUSTACHE DESCHAMPS, op. cit., t. II, p. 29.

${ }^{36}$ Ibid., t. III, p. 100.

37 Guillaume de Machaut, La Prise d'Alexandrie ou Chronique du roi Pierre ${ }^{e r}$ de Lusignan, éd. M. L. DE MAS LATRIE, Genève, Fick, 1877, v. 7182-7185 et v. 8851-8854; à ce sujet, voir Jacqueline CERQUIGLINI, art. cit., p. 37.

${ }^{38}$ Roger S. LoOmIS, Verses on the Nine Worthies, Modern Philology, XV, 4, 1917, p. 19.

39 Au sujet de Louis de Laval, voir le chapitre que Frédéric DUVAL lui consacre dans La traduction du Romuleon, Genève, Droz, 2001, p. 217-239.
} 
de Laval avait épousé Bertrand du Guesclin, et ses deux frères, Guy XIV comte de Laval et André de Lohéac, avaient combattu aux côtés de Jeanne d'Arc. On constate donc une volonté d'accroître la gloire d'un lignage ou en tout cas de signaler de manière indirecte une proximité avec les grands personnages du temps ${ }^{40}$, qui viennent constituer une généalogie idéale et merveilleuse des princes.

Les trois compilations sur les Preux qui nous sont parvenues présentent dans leur projet une problématique un peu différente. Ces volumineuses compilations, datant de la fin du Moyen Âge, se présentent comme une succession de biographies « historiques » des Preux, vraisemblablement dans le but de remplir à nouveau la structure vide de la liste. Cette volonté historique est visible dans un bouleversement de l'ordre des triades, Josué ouvrant l'Histoire des Neuf Preux et le Triomphe ${ }^{41}$. Dans le prologue de Sébastien Mamerot se dégagent deux objectifs, d'une part rendre accessible en un volume un savoir dispersé dans de nombreux livres et d'autre part, donner une version véridique des hauts faits des Preux, ordonnée «selon les temps de leurs regnes et chevaleries » (fol. 1, ÖNB, ms. 2577). Il se plie en cela aux exigences de son commanditaire, Louis de Laval, qui veut empêcher que «les bons chevaliers et autres nobles par telle tediacion ne delaissent depuis cy en avant enquerir, serchier et sçavor tant nobles fais que par raison selon toutes loables meurs ne doivent ygnorer»(fol. 1d, ÖNB, ms. 2577). Les Preux sont donc les jalons d'une histoire qui garde la mémoire des hauts faits accomplis afin de donner au lecteur envie d'émuler ces exploits. Les intentions de Sébastien Mamerot sont

\footnotetext{
40 Helena KogEN, «Les goûts littéraires de la famille de Laval: constitution d'une bibliothèque familiale », dans Le goût du lecteur à la fin du Moyen Âge, Cahiers du Léopard d'or, 11, 2006, p. 213-223.

${ }^{41}$ Traditionnellement, la triade païenne ouvre la série des Preux, suivie de la triade biblique puis de la triade chrétienne, esquissant une histoire du salut dans une progression eschatologique.
} 
proches ici de celles qu'exprime Jean Froissart dans le prologue des Chroniques $^{42}$ :

« Or doient donc tout jone gentil homme, qui se voellent avancier, avoir ardant desir d'acquerre le fait et le renommée de proèce, par quoi il soient mis et compté ou nombre des preux [...]. Je sui seurs que, se ilz regardent et lisent en ce livre, que il trouveront otant de grans fais et de belles apertises d'armes, de durs rencontres, de fors assaus, de fières batailles et de tous autres maniements d'armes qui se descendent des membres de proèce, que en nulle hystore dont on puist parler, tant soit anchiienne ne nouvelle. Et ce sera à yaus matère et exemples de yaux encoragier en bien faisant, car la memore des bons et li recors des preux atisent et enflament par raison les coers des jones bacheliers, qui tirent et tendent à toute perfection d'onneur, de quoi proèce est li principaux chiés et li certains ressors. "

Cependant, du fait de l'ampleur de chaque section, la vision donnée de chaque Preux est moins tranchée, plus nuancée et peut laisser une certaine place à la critique. Aussi Sébastien Mamerot évoque-t-il la cruauté d'Alexandre en ces termes :

« Orose dit d'Alexandre, combien qu'il feust tenu pour courtoys et large, qu'il ne fut pas moins crueulx a ses parens et a ses amys que a ses ennemys. » (fol. 184a, ÖNB, ms. 2577)

Cette exigence d'historicité est visible dans le choix des sources, qui va pousser Sébastien Mamerot à suivre des textes historiques et des chroniques, tout en faisant parfois des concessions à une certaine vulgate héritée des romans. La même exigence se retrouve, du moins partiellement, chez l'auteur du Triomphe des Neuf Preux qui, pour la section consacrée à Alexandre, utilise une version abrégée de la Vie d'Alexandre de Plutarque, certainement par l'intermédiaire d'une traduction latine ${ }^{43}$, attitude proche de celle qu'adopteront les Humanistes.

\footnotetext{
${ }^{42}$ JEAN FROISSART, op. cit., p. 2-3

${ }^{43}$ David J. A. Ross, Alexander Historiatus, Frankfurt am Main, Athenäum, 1988, p. 126 (1 ${ }^{\text {ère }}$ édition : Londres, Université de Londres, 1963).
} 
Si le motif des Preux a une vocation exemplaire incontestable et a connu de nombreux emplois moralisateurs qui ont été étudiés en relation avec le thème de l'Ubi sunt ${ }^{44}$, il n'en demeure pas moins qu'il comporte une ambiguïté fondamentale. Pour exceptionnels qu'ils fussent, les Preux n'en étaient pas moins mortels, et il n'y pas loin de l'honneur à l'orgueil. Ils ont ainsi pu être utilisés comme illustration des mutacions de Fortune; on retrouve d'ailleurs plusieurs des Preux dans le De casibus virorum illustrium ${ }^{45}$ de Boccace, dont le succès a été immense en France par l'intermédiaire de la traduction de Laurent de Premierfait, ou encore dans le Livre de la Mutacion de Fortune ${ }^{46}$ de Christine de Pizan. Cette tension n'apparaît nulle part aussi clairement que chez Thomas III de Saluces dans le Chevalier Errant ${ }^{47}$. Dans sa quête à la recherche de sa dame, le Chevalier arrive chez Fortune; les Preux apparaissent alors à deux reprises. Des plaignants, menés par Penthésilée, viennent exposer leur cas à Fortune et déplorent leur gloire passée; parmi eux se trouvent Hector, Alexandre, Jules César, Arthur et Judas Machabée. Le Chevalier rencontre le reste des Preux et des Preuses dans le Palais aux Élus où se trouvent dix-huit sièges qui leur sont réservés. Les Preux, personnages complexes et présentant une grande richesse dans leurs représentations, ont ainsi pu être utilisés comme exempla bonorum ou comme exempla malorum; la structure canonique de la liste des Neuf Preux ne suffit pas, en les subsumant, à faire perdre toute substance aux héros individuels, qui illustrent pour la plupart aussi bien la vaillance et la gloire individuelle que l'aspect transitoire de l'existence humaine et les retournements de la fortune.

\footnotetext{
${ }^{44}$ Voir Gianni Mombello, art. cit et Tania VAN Hemelryck « Où sont les "Neuf Preux"? Variations sur un thème médiéval », Studi francesi, 1998, p.1-8.

45 Giovanni Boccaccio, De casibus virorum illustrium, éd. Pier Giorgio Ricci et Vittorio ZACCARIA, Tutte le opere, éd. par Vittore BRANCA, vol. IX, Milan, Mondadori, 1983.

${ }^{46}$ Christine De PisAn, Le livre de la Mutacion de Fortune, éd. Suzanne Solente, Paris, Picard, 1959.

${ }^{47}$ Voir l'analyse que fait de ce texte Richard TRACHSLER dans Disjointures - Conjointures, p. 325-365.
} 
Si l'on ne s'étonne pas aujourd'hui de voir sur nos cartes à jouer le roi de cœur sous les traits de Charlemagne, de voir Jules César en roi de carreau, David en roi de pique, Alexandre en roi de trèfle ou Hector en valet de carreau, ainsi réunis, ils ne nous évoquent plus le groupe des Neuf Preux. Ce motif va connaître un déclin parallèle à celui de la chevalerie ; intimement lié à l'idéal chevaleresque entretenu dans les cours de l'époque, il ne lui survivra pas. Même si ces figures ont conservé leur popularité et leur renom, la transmission des faits les concernant s'est faite individuellement et selon des modalités différentes pour chaque héros. 